Article

\title{
High-Precision Attitude Post-Processing and Initial Verification for the ZY-3 Satellite
}

\author{
Xinming Tang ${ }^{1,2}$, Junfeng Xie ${ }^{1, *}$, Xiao Wang ${ }^{3,4, *}$ and Wanshou Jiang 5
}

1 Satellite Surveying and Mapping Application Center, NASG, 28 Lianhuachi West Road, Beijing 100830, China; E-Mail: Txm@sasmac.cn

2 Jiangsu Center for Collaborative Innovation in Geographical Information Resource Development and Application, 1 Wenyuan Road, Nanjing 210023, China

3 Institute of Remote Sensing and Digital Earth Chinese Academy of Sciences, 3 Datun Road, Beijing 100101, China

4 Beijing UniStrong Science \& Technology Co., Ltd., Beijing 100015, China

5 State Key Laboratory of Information Engineering in Surveying, Mapping and Remote Sensing, Wuhan University, 129 Luoyu Road, Wuhan 430079, China; E-Mail: jws@whu.edu.cn

* Authors to whom correspondence should be addressed:

E-Mails: xiejf@sasmac.cn (J.X.);wangxiao@radi.ac.cn (X.W.); Tel.: +86-10-6480-6207 (X.W.).

Academic Editors: Richard Müller and Prasad S. Thenkabail

Received: 13 October 2014 / Accepted: 5 December 2014 / Published: 24 December 2014

\begin{abstract}
Attitude data, which is the important data strongly correlated with the geometric accuracy of optical remote sensing satellite images, are generally obtained using a real-time Extended Kalman Filter (EKF) with star-tracker and gyro data for current high-resolution satellites, such as Orb-view, IKONOS, Quickbird,Pleiades, and ZY-3.We propose a forwardbackward Unscented Kalman Filter (UKF) for post-processing, and the proposed method employs UKF to suppress noise by using an unscented transformation (UT) rather than an EKF in a nonlinear attitude system. Moreover, this method makes full use of the collected data in the fixed-interval and computational resources on the ground, and it determines optimal attitude results by forward-backward filtering and weighted smoothing with the raw star-tracker and gyro data collected for a fixed period. In this study, the principle and implementation of the proposed method are described. The post-processed attitude was compared with the on-board attitude, and the absolute accuracy was evaluated by the two methods. One method compares the positioning accuracy of the object space coordinates with the post-processed and on-board attitude data without using ground control points
\end{abstract}


(GCPs). The other method compares the tie-point residuals of the image coordinates after a free net adjustment. In addition, the internal and external parameters of the camera were accurately calibrated before use for an objective evaluation of the attitude accuracy. The experimental results reveal that the accuracy of the post-processed attitude is superior to the accuracy of the on-board processed attitude. This method has been applied to the ZiYuan-3 satellite system for processing the raw star-tracker and gyro data daily.

Keywords: attitude accuracy; star tracker/gyro; post-processing; geometric accuracy

\section{Introduction}

Satellite attitude is used in real-time support control and other decision functions, and it also plays an important role in locating objects in remote sensing satellite images [1,2].Although the ground location error of the satellite image would be influenced by many factors such as the camera parameters and orbit position et al., the attitude error is one of the main error sources. The ground location error caused by 1 arc s attitude error will be approximately $2.5 \mathrm{~m}$ for optical satellites from a nominal altitude of $504 \mathrm{~km}$. Currently, to improve attitude accuracy and stability, most high-resolution remote sensing satellites are equipped with several star trackers and gyros. Star tracker, which has the highest accuracy and no drift, is used to obtain absolute inertial attitude with low frequency. Gyros can measure the angular velocity with high frequency. The combined attitude determination with star tracker and gyro data often uses filtering technology to produce the optimal result, i.e., better results than when using a single attitude sensor. Therefore, the filter design and filtering method selection play a vital role in attitude output accuracy.

The Kalman Filtering (KF) theory was first proposed by R. E. Kalman in the 1960s [3]. The theory greatly promoted the development of navigation technology combined with star tracker and gyro capabilities. KF is simple and used real-time, and it can obtain the least square estimation of a system state for a linear system with Gaussian noise distribution. However, KF has shortcomings, such as a large amount of computations and poor fault tolerance when applied to a high-dimensional system. A number of strategies have been proposed to increase the robustness of KF methodologies [4-6]. To apply the KF theory to a nonlinear system, an Extended Kalman Filter (EKF)was proposed by Anderson and Moore [7]. The EKF is a suboptimal filter that linearizes and filters a nonlinear system. For a nonlinear system, the performance of EKF is limited, mainly due to the truncation of all but the first-order terms [8-10].To reduce the linearization error and increase the stability of EKF, several improved algorithms have been proposed, such as iterative filtering and second-order Taylor expansion, Cholesky decomposition, upper triangular factor (U) and diagonal factor (D) (U-D factorization), and triangularization algorithms [11-16].However, these improved EKF methods cannot solve the essential problems of linearization error.

The Unscented Kalman filter (UKF) was proposed by Julier and Uhlmann [17]. UKF employs deterministic sampling theory to the propagation of Gauss Random Variables (GRV) in nonlinear equations, rather than linearizing the nonlinear system $[18,19]$. The distribution of state variables is approximated by GRV, and GRV is described by a sample point set selected by the square root decomposition method. The mean and variance of the GRV can be accurately calculated by weighting 
these sample points. When GRV is propagated in nonlinear systems, the accuracy of the mean and variance of GRV is similar to that of the second-order Taylor expansion, while the EKF method can achieve first-order Taylor expansion accuracy. The performance of UKF is superior when compared with EKF [20,21].The square root UKF was proposed by Wan and Merwe to guarantee that the state covariance matrix has positive semi-definiteness and reduce the computational requirements [22]. Overall, the estimation accuracy of UKF is improved, to an extent, compared with that of EKF when the system has nonlinear characteristics.

Generally, EKF is used in real-time satellite attitude determination to obtain fast, robust attitude data and maintain the normal functions of the attitude control system. The attitude of the high-resolution remote sensing satellites, such as OrbView, IKONOS, and SPOT, was determined by using multiple star trackers and gyros with EKF [23-25]. The attitude accuracy using the real-time filtering is sacrificed to satisfy the time limitations. KF, EKF and UKF are used for batch estimations to produce more accurate attitudes [26-28]. The fixed-interval smoother based on KF was proposed by Rauch-Tung-Striebel in 1965 [26] and was later developed [27]. The forward filter first processes the measurements; then, a separate backward smoother is used to obtain the smoothing result. The smoother based on EKF has been proposed and tested [28], and it includes three components, i.e., a forward filter, a backward filter, and a separate smoother that combines these results. The posteriori batch filters involve a post-analysis of the state estimation related to the observation data, and they often yield an accuracy superior to that of the sequential filter. Fora few high-resolution remote sensing satellites, such as Landsat-7, GLAS, and ALOS, the raw data of the star trackers and gyros are recorded and downlinked to the ground station for posteriori batch filtering [28-30].

As described previously, UKF is an attractive option for spacecraft due to its ability to estimate nonlinearities accurately. Moreover, a batch filter can obtain higher attitude accuracy than sequential filters. Therefore, the main goal of this study is to propose a forward-backward Unscented Kalman Filter (UKF). This method is developed based on the standard UKF algorithm [18-21]. The innovation of this approach is in employing a forward-backward smoothing strategy, which takes the accumulated raw star tracker and gyro data in an interval of time as its input data, the global optimal estimation is weight averaged with forward and backward state estimation based on their covariances. This approach is deployed for the ZiYuan-3(ZY-3) satellite's post-attitude determination.ZY-3 is the first Chinese civilian high-resolution stereo-mapping satellite, which was launched on 9 January 2012. The ZY-3 satellite carries three panchromatic cameras (forward-view, nadir-view, and backward-view), for panchromatic images the ground sampling distance (GSD) of the nadir-view camera is $2.1 \mathrm{~m}$ and that of forwardview and backward-view are both $3.5 \mathrm{~m}$. The size of CCD detector of forward-view and backward-view cameras is $10 \mathrm{~mm} \times 10 \mathrm{~mm}$, and nadir-view camera's is $7 \mathrm{~mm} \times 7 \mathrm{~mm}$. The focal length of the camera is $1700 \mathrm{~mm}$. These three panchromatic cameras form a triple-linear array for stereo topographic mapping at 1:50,000 scale and updating topographic maps at 1:25,000 scale [31,32]. The satellite is equipped with three star trackers (two ASTRO-10 star trackers and a domestic APS star tracker), three group-gyros and multiple infrared Earth trackers (among other sensors). The raw attitude data from the star tracker and gyro are downlinked for ground-based attitude post-processing to improve the accuracy.

This study demonstrates the application of the proposed method in the attitude estimation of ZY-3 based on gyro and star-tracker measurements. A comparison between the post-processed and on-board results is also presented. The accuracy of the post-processed attitude result was evaluated by comparing it 
with the on-board attitude. First, the difference between the post-processed and on-board attitude was given. Next, the absolute accuracy was assessed using two methods. One method compares the positioning accuracy of the object space coordinates with the post-processed and on-board attitude data without using GCPs. The other method compares the tie point residuals of the image coordinates after a free net adjustment. In addition, to evaluate objectively the attitude accuracy in this study, the internal and external parameters of the camera were accurately calibrated before use. The results reveal that the accuracy of the post-processed attitude is better than the accuracy of the on-board processed attitude.

This paper is organized as follows. The filtering principle of UKF is formulated in Section 2. Section 3 compares the estimation accuracy of the post-processed attitude and the on-board processed attitude. The experiment results of ZY-3 are presented. Finally, a summary is provided in Section 4.

\section{Methodology}

\subsection{Principle}

The principle of the proposed forward-backward UKF is shown in Figure 1. The method includes forward UKF, backward UKF and covariance weighted smoothing. First, the UKF filtering is implemented from the front to the back, and the filtering result is recorded. Second, UKF filtering is executed in the reverse direction. The two filtering processes are independent, and the smoothing is performed based on the last two filtering results. The given threshold value determines whether the iteration convergence process stops. To understand the proposed filtering strategy, the filtering process of UKF, which is the foundation of the proposed method, is briefly introduced in advance.

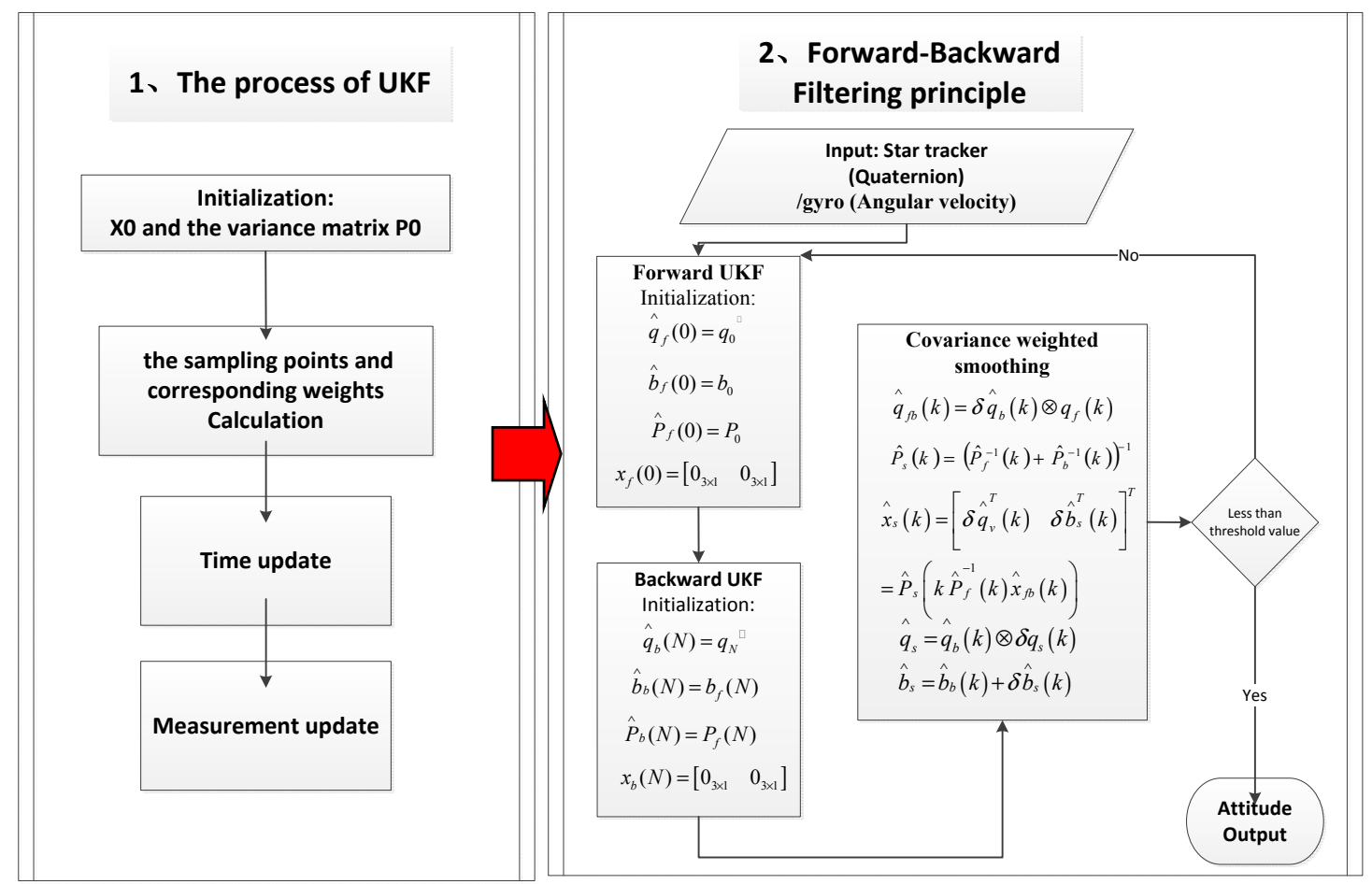

Figure 1.The principle of the forward-backward Unscented Kalman Filter (UKF). 


\subsubsection{The UKF Filtering Process}

The proposed algorithm for all of the filters explained here begins with the definition of the state $(x)$ and measurement $(y)$ systems. The dynamic and measurement equations are presented as follows:

State equation:

$$
\dot{x}_{k}=F\left(t_{k}\right) x_{k}+G\left(t_{k}\right) w_{k}
$$

Observation equation:

$$
\begin{aligned}
& y_{k}=h\left(x_{k}, t_{k}\right)+V_{k} \\
& \dot{x}_{k}=F\left(t_{k}\right) x_{k}+G\left(t_{k}\right) w_{k}
\end{aligned}
$$

where $W_{k}$ and $V_{k}$ are the process and measurement noise vectors, which are assumed to have a Gaussian white noise of zero. $F\left(t_{k}\right)$ is the dynamic model function, and $h\left(x_{k}, t_{k}\right)$ is themeasurementmodel function.

Figure 2 shows the flow chart of UKF. The initial state vector $\mathbf{X}_{0}$ and the variance matrix $\mathbf{P}_{0}$ is given. The basic steps are shown below according to the state equation and the measurement equation [21].

The symbols - and ^ represent the mean and estimated values, respectively.

(1) Initialization:

$$
\hat{\mathbf{x}}_{0}=E\left[\mathbf{x}_{0}\right], \mathbf{P}_{0}=E\left[\left(\mathbf{x}_{0}-\hat{\mathbf{x}}_{0}\right)\left(\mathbf{x}_{0}-\hat{\mathbf{x}}_{0}\right)^{T}\right]
$$

(2) Calculation of the sampling points and corresponding weights:

The state dimension $n=6$, with $2 n+1$ sampling points and the corresponding weights are calculated when $k \geq 1$ and $i=1,2, \ldots, n$.

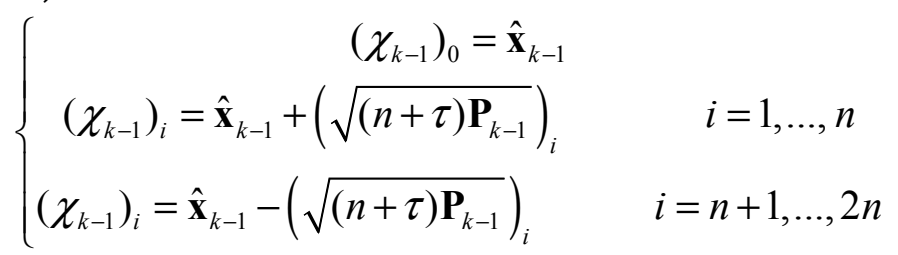

$\sqrt{(n+\tau) P_{k-1}}$ is the $i$ th column of the matrix square root of $(n+\tau) P_{k-1}$. Note that the matrix of $\sqrt{(n+\tau) P_{k-1}}$ is assumed to be symmetric and positive-definite and can be obtained by the square root using the Cholesky decomposition.

$$
\begin{gathered}
W_{m i}=\left\{\begin{array}{lc}
\frac{\tau}{(n+\tau)} & i=0 \\
\frac{1}{[2(n+\tau)]} & i=1, \ldots, 2 n
\end{array}\right. \\
W_{c i}=\left\{\begin{array}{lc}
\frac{\tau}{(n+\tau)}+1-\alpha^{2}+\beta & i=0 \\
\frac{1}{[2(n+\tau)]} & i=1, \ldots, 2 n
\end{array}\right.
\end{gathered}
$$

$W_{m i}$ and $W_{c i}$ are the weighting factors of the mean value and the variance of the state parameters, $\mathbf{X}$, and $\alpha$ is the distribution of the sampling points around the state mean. Small positive definite values $0 \leq \alpha \leq 1$ are often used to avoid nonlocal effects of the sampling point due to the nonlinearity of the state equation. 


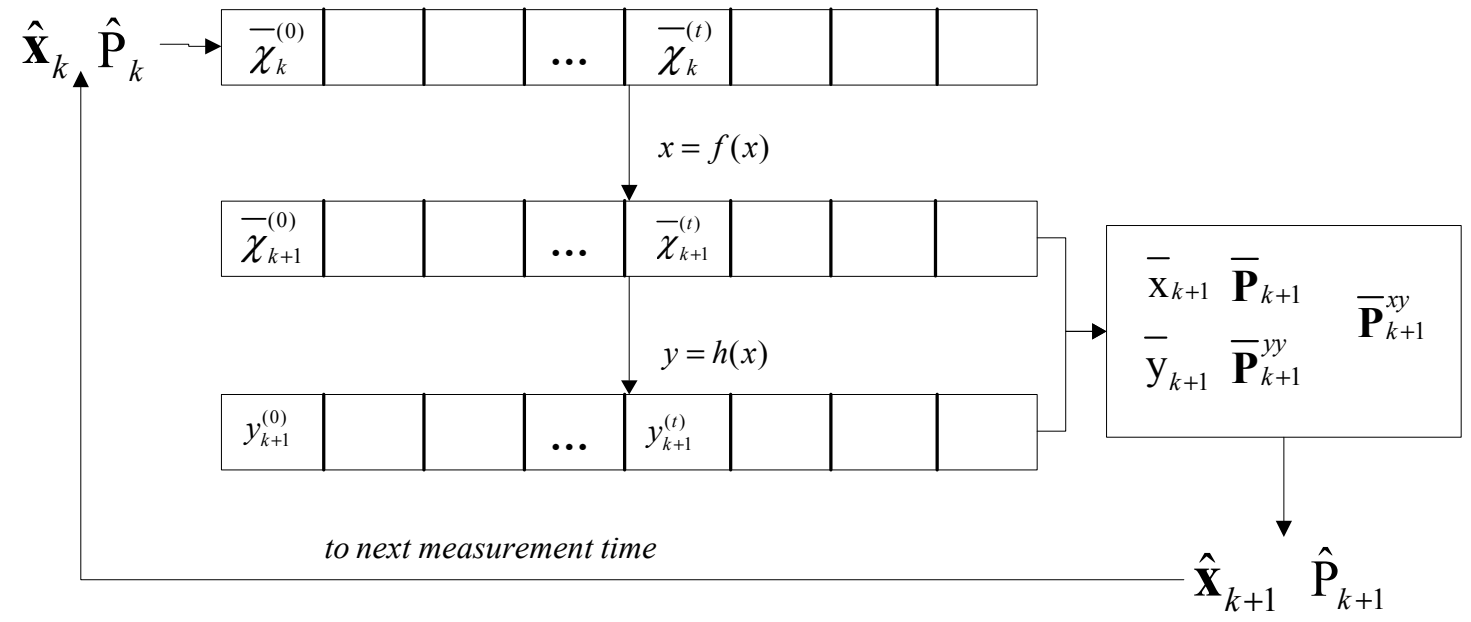

Figure 2. Unscented Kalman Filtering.

Parameter $\beta$ plays a role in incorporating the prior knowledge of the distribution of $x$. When $\beta=2$, a Gaussian distribution of $x$ is optimal, which is otherwise determined by a simulation experiment. The value of $\tau$ is the scalar parameter. When the state value is univariable, $\tau=\alpha^{2}(n+2)-n$, and when the state value ismultivariable, $\tau=3 \alpha^{2}-n$.

(3) Time update:

$$
\begin{gathered}
\chi_{k / k-1}=f\left(\chi_{k-1}, k-1\right), \hat{x}_{k}^{-}=\sum_{i=0}^{2 n} W_{m i} \chi_{i, k / k-1} \\
P_{k}^{-}=\sum_{i=0}^{2 n} W_{c i}\left[\chi_{i, k / k-1}-\hat{x}_{k}^{-}\right]\left[\chi_{i, k / k-1}-\hat{x}_{k}^{-}\right]^{T}+\mathrm{Q}_{k} \\
y_{k / k-1}=H\left(\chi_{k / k-1}, k\right), \hat{y}_{k}^{-}=\sum_{i=0}^{2 n} W_{m i} y_{i, k / k-1}
\end{gathered}
$$

The sigma points are propagated through the dynamic model. $f\left(\chi_{k-1}, k-1\right)$ is the discrete function of $F\left(t_{k-1}\right) . F\left(t_{k-1}\right)$ can be found in Equation (1).

(4) Measurement update:

$$
\begin{aligned}
& P_{\hat{y}_{k} \hat{y}_{k}}=\sum_{i=0}^{2 n} W_{c i}\left[y_{i, k \mid k-1}-\hat{y}_{k}^{-}\right]\left[y_{i, k \mid k-1}-\hat{y}_{k}^{-}\right]^{T}+\mathbf{R}_{k} \\
& P x_{k} y_{k}=\sum_{i=0}^{2 n} W_{c i}\left[\chi_{i, k \mid k-1}-\hat{x}_{k}^{-}\right]\left[y_{i, k \mid k-1}-\hat{y}_{k}^{-}\right]^{T} \\
& K_{k}=P_{x_{k} y_{k}} P_{\hat{y}_{k} \hat{y}_{k}}^{-1} \\
& \hat{x}_{k}=\hat{x}_{k}^{-}+K_{k}\left(y_{k}-\hat{y}_{k}^{-}\right) \\
& P_{k}=P_{k}^{-}-K_{k} P_{\hat{y}_{k} \hat{y}_{k}} K_{k}^{T}
\end{aligned}
$$

In Equations (8) and (10), $Q_{k}$ and $R_{k}$ are the covariance matrices of the state and measurement noises, respectively.

Once the state vector $\hat{x}_{k}=\left[\delta q_{v}^{T} \delta b^{T}\right]^{T}$ is calculated using Equation (13), the estimated quaternion and gyro biases at the current measurement epoch $t_{k}$ can be obtained as follows: 


$$
\begin{gathered}
\hat{q}=2 \delta \hat{q} \otimes q^{\sim} \\
\hat{b}=b^{\sim}+\delta b
\end{gathered}
$$

where $q^{\sim}$ and $b^{\sim}$ are the predicted values at time $t_{k}$ based on the observation at time $t_{k-1}$.

\subsubsection{Forward-Backward Filtering Strategy}

A forward-backward smoother with UKF was designed based on previous concepts [28]. This strategy uses the two-direction iterative approximation. Each filtering process includes a forward UKF, a backward UKF, and a covariance weighted smoothing. The smoothed estimates were obtained by averaging the forward and backward state estimates with weights based on their covariances. This approach minimizes the covariances of the averaged estimates.

\section{(1) Forward UKF}

The suffixes $f, b$, and $s$ represent forward filtering, backward filtering, and smoothing, respectively.

In the forward filtering process, the initial estimated value $\hat{q}_{f}(0)$ is obtained by dual vector determination with the star tracker observation at the first measurement epoch $t$. The initial estimated value of the gyro bias $\hat{b}_{f}(0)$ is $b_{0}$, which can be analyzed using the down linked telemetry data. In addition, the initial values of the error covariance $\hat{P}_{f}(0)$ are also obtained from the empirical value of $P_{0}$, which is derived from the star tracker/gyro measurements.

$$
\hat{q}_{f}(0)=q_{0}{ }^{-}, \hat{b}_{f}(0)=b_{0}, \hat{P}_{f}(0)=P_{0}
$$

The initial estimated state variable is

$$
x_{f}(0)=\left[\begin{array}{ll}
0_{3 \times 1} & 0_{3 \times 1}
\end{array}\right]
$$

Forward filtering was executed according to the working flow described in the previous section, and begins at an initial time of $t_{0}$ and ends at the last measurement epoch, $t_{\mathrm{N}}$. For the ZY-3 satellite, the output of two star trackers and three gyros are used as the observation values for the filter. Their sampling time is completely synchronous with the same frequency $(4 \mathrm{~Hz})$ and the sampling interval is $0.25 \mathrm{~s}$. When the sigma points are selected using Equation (4), each sigma point is propagated to all measurement epochs using Equation (7). The measurements for each sigma point are calculated using Equation (9). The star tracker/gyro measurements are updated to ensure optimal gain, and the covariance matrix $P_{f}(k)$, the estimated drift $b_{f}(k)$ and the estimate at each measurement epoch time, $k$, are recorded for covariance weighted smoothing. The estimate quaternion $\hat{q}_{f}$ and drift $\hat{b}_{f}$ in the forward filtering process are corrected with state vector $\hat{x}_{k}$ using Equations (15) and (16).

(2) Backward UKF

Backward filter processing is similar to forward processing. However, forward filtering proceeds from time $t_{0}$ to time $t_{N}$ with increasing time, while backward filtering proceeds from time $t_{N}$ to $t_{0}$ with decreasing time.

For backward filtering, the initial estimated value of $\hat{q}_{b}(N)$ is the star tracker observations $q_{N}^{\sim}$ at thelast measurement epoch $t_{\mathrm{N}}$, and the initial estimated value of the gyro bias $\hat{b}_{b}(N)$ and error covariance $\hat{P}_{b}(N)$ are obtained from the forward filtering results.

$$
\hat{q}_{b}(N)=q_{N} \sim, \hat{b}_{b}(N)=b_{f}(N), \hat{P}_{b}(N)=P_{f}(N)
$$


The selection of the initial state variable value $x_{b}(N)$ is the same as the selection of $x_{f}(0)$.

The state variable $x_{b}(N)$ at the last measurement epoch $t_{\mathrm{N}}$ is

$$
x_{b}(N)=\left[\begin{array}{ll}
0_{3 \times 1} & 0_{3 \times 1}
\end{array}\right]
$$

In the backward filtering process, the observed value is updated in the reverse direction. This process is the same as the forward filtering process. The covariance matrix $P_{b}(k)$ and drift $b_{b}(k)$ at each measurement epoch time $k$ are recorded for the covariance weighted smoothing.

(3) Covariance Weighted Smoothing

When the forward and backward filtering are finalized, smoothed estimates are obtained by averaging the forward state and backward state estimates with their weights based on their covariance. This step minimizes the covariance of the averaged estimates. For the covariance weighted smoothing, the following algorithm is given:

$$
\begin{gathered}
\hat{q}_{f b}(k)=\delta \hat{q}_{b}(k) \otimes q_{f}(k) \\
\hat{P}_{s}(k)=\left(\hat{P}_{f}^{-1}(k)+\hat{P}_{b}^{-1}(k)\right)^{-1} \\
\hat{x}_{s}(k)=\left[\delta \hat{q}_{v}^{T}(k) \quad \delta \hat{b}_{s}^{T}(k)\right]^{T}=\hat{P}_{s}\left(k \hat{P}_{f}^{-1}(k) \hat{x}_{f b}(k)\right) \\
\hat{q}_{s}=\hat{q}_{b}(k) \otimes \delta q_{s}(k) \\
\hat{b}_{s}=\hat{b}_{b}(k)+\delta \hat{b}_{s}(k)
\end{gathered}
$$

The smoothing filter procedure is shown in Figure 2. If the following condition is not satisfied, the filter will continue from the forward UKF with the previous covariance weighted smoothing result [33]:

$$
\left|\frac{R M S_{\text {new }}-R M S_{\text {old }}}{R M S_{\text {old }}}\right|<\varepsilon
$$

where $\varepsilon$ is a pre-set value, and the root mean square (RMS) of the observation residuals is computed as follows [34]:

$$
R M S=\left\{\frac{\sum_{i=1}^{N} y_{i}^{T} R_{i}^{-1} y_{i}}{m}\right\}
$$

here, $N$ is the total measurement time, and $R_{i}$ and $y_{i}$ are the measurement noise covariance and the measurement residual at time $t_{i}$, respectively. In addition, $m$ is the total number of measurements.

\subsection{Workflow}

The experiment workflow is shown in Figure 3, which includes the forward-backward filtering and accuracy evaluation. The proposed forward-backward filtering process includes raw attitude-data unpacking, outlier identification and rejection, forward UKF, backward UKF and covariance-weighted smoothing. Before filtering, the outliers in the star tracker and gyro data that are caused by channel transmission problems or other errors should be removed to avoid divergent attitude filtering results 
during the global attitude estimation process; then, the raw star-tracker and gyro data processing is accomplished using a forward-backward smoother with UKF.

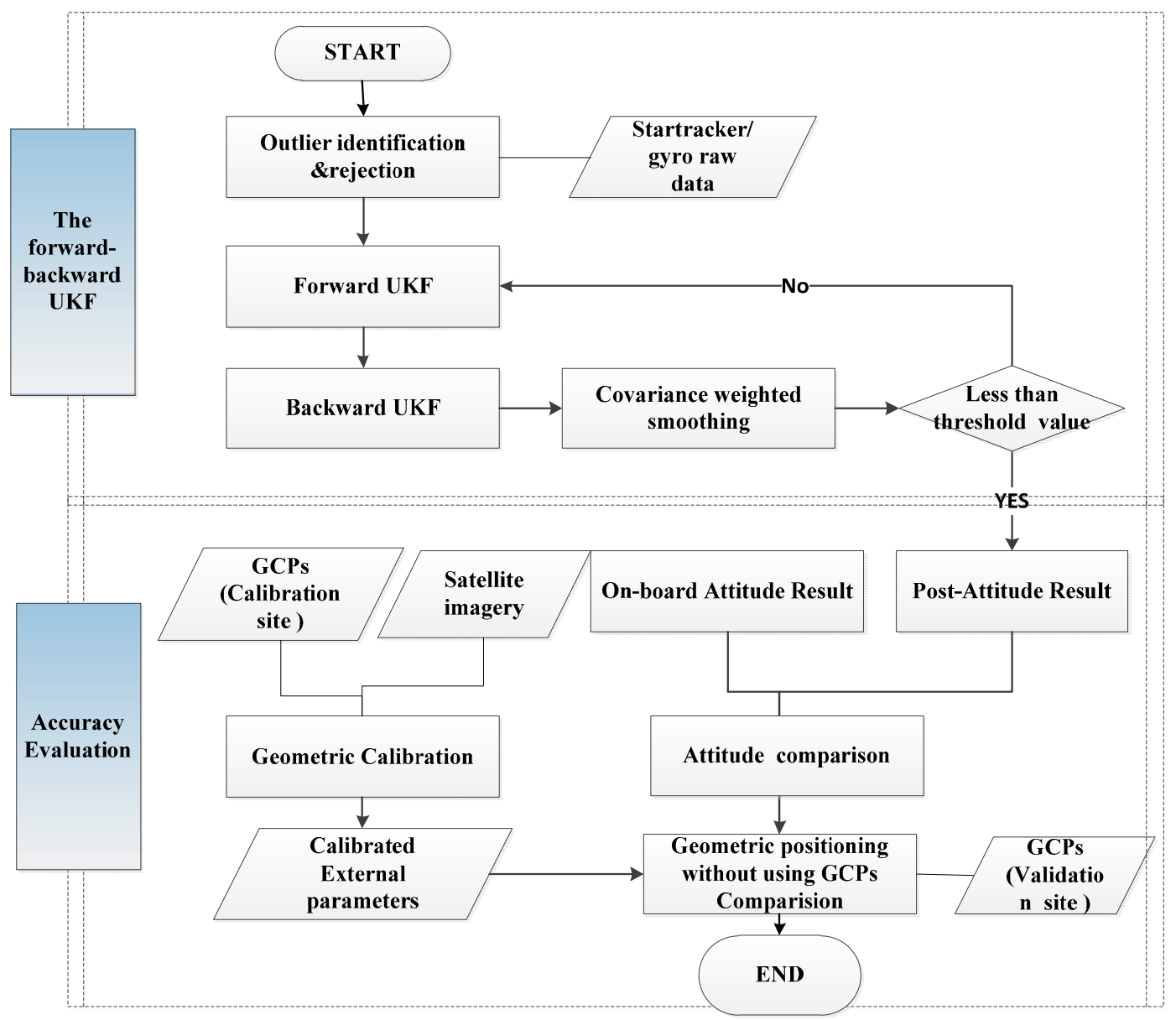

Figure 3. The attitude post-processing and accuracy verification workflow.

The accuracy evaluation process includes rigorous geometric imaging model construction, geometric calibration and geometric accuracy comparisons without the use of GCPs. To evaluate the post-processed and on-board attitude accuracy, on-orbit geometric calibration of the camera parameters is required. Although the camera parameters were accurately calibrated in the laboratory before the satellite was launched, they may change due to the intense vibrations that occur during launching or changes in the environmental factors in space, such as temperature and atmospheric pressure. The rigorous imaging model is first constructed; then, the geometric calibration model is built for the on-orbit camera parameter solution. GCPs are used as references for calibration and accuracy evaluations. The details are given below.

\subsubsection{The Forward-Backward Filtering for Attitude Post-Processing}

Generally, multiple attitude sensors are loaded to obtain attitude information. The proposed filtering method is performed with the raw data of two star trackers and three gyros in the following steps.

(1) Outlier identification for the unpacked raw star tracker/gyro data. For the raw gyro data: firstly, determine whether the gyro output exceeds the measurement range. If yes, the gyro output is 
judged as outlier. If not, the angle increment output of gyro output in current period should be compared with that in the last period, these output would be considered as outliers when the measured angle increment is larger than the theoretical value. For those raw star tracker data: transform the star tracker output (quaternion) to the measurement direction vectors defined in the optical axis and horizontal axis, and then fit these vectors using a polynomial approach. If the deviation of the measured and fitted values is larger than the threshold value, the measured values will be considered as outliers and excluded.

(2) Determine the initial estimated $\hat{q}_{f}(0)$ in the forward UKF by the dual-vector attitude determination method with the initial output of two star trackers [35], and set the initial value of the state variable and gyro bias using Equations (17) and (18). The initial gyro bias $\hat{b}_{f}(0)$ of the real-time processing result is recorded and transferred to a ground station. The sampling points and corresponding weights are obtained by Equations (4)-(6). The error covariance $\hat{P}_{f}(0)$ sets an empirical value by analyzing the real-time measurement results. The time and measurement are updated by Equations (7)-(14). Once the state vector $\hat{x}_{k}=\left[\delta q_{v}^{T} \delta b^{T}\right]^{T}$ at epoch $t_{k}$ is calculated with Equation (13), the estimated quaternion and gyro biases at epoch $t_{k}$ are updated by Equations (15) and (16).

(3) The backward filtering is implemented from time $t_{N}$ to $t_{0}$ with decreasing time. The initial value $\hat{q}_{b}(N)$ is obtained by the dual-vector determination with the two star trackers' output at the last measurement epoch $t_{\mathrm{N}}$. The initial value of the gyro bias $\hat{b}_{b}(N)$ and error covariance $\widehat{P}_{b}(N)$ can bedirectly obtained from the forward filtering results. All initial values are set by Equations (19) and (20).Similarly, the time and measurement are updated by Equations (7)-(14). Once the state vector $\hat{x}_{k}=\left[\delta q_{v}^{T} \delta b^{T}\right]^{T}$ is calculated by using Equation (13), the estimated quaternion and gyro biases at the epoch $t_{k}$ are updated by Equations (15) and (16).

(4) Record the covariance $\hat{P}_{f}(k)$ and $\hat{P}_{b}(k)$ in the forward and backward filtering processes, and calculate the smoothing covariance $\hat{P}_{s}(k)$ from Equation (22). The smoothing state variables $\hat{x}_{s}(k)$ can be calculated by Equation (23); then, the smoothing estimated values of $\hat{q}_{s}$ and $\hat{b}_{s}$ can be updated by Equations (24) and (25). The previous and current RMS values of the observation residuals are calculated from Equation (27). A pre-set value $\varepsilon$ is set to $1 \times 10^{-3}$. If the difference between the new RMS and old RMS is less than $\varepsilon$, then the iteration process stops.

\subsubsection{Attitude Accuracy Comparison and Evaluation}

The rigorous imaging model establishes the relationship between image space and object space. Based on this model, the geometric calibration and attitude accuracy evaluation can be performed.

(1) Construct a rigorous geometric imaging model of the ZY-3 satellite, as shown in Equation (28).This imaging model borrows concepts from SPOT-5 [36], and it is sufficiently validated [37,38].

$$
\left[\begin{array}{c}
X \\
Y \\
Z
\end{array}\right]_{W G S 84}=\left[\begin{array}{c}
X_{G P S} \\
Y_{G P S} \\
Z_{G P S}
\end{array}\right]_{W G S 84}+m^{\prime} R_{J 2000}^{W G S 84} R_{\text {star }}^{J 2000} R_{\text {camera }}^{\text {body }}\left(\begin{array}{l}
\operatorname{tg}\left(\psi_{Y}^{\prime}\right) \\
\operatorname{tg}\left(\psi_{X}^{\prime}\right) \\
-1
\end{array}\right)
$$


where $\operatorname{tg}\left(\psi_{Y}{ }^{\prime}\right), \operatorname{tg}\left(\psi_{X}{ }^{\prime}\right)$ are the line-of-sight vectors for each CCD detector in the camera's coordinate system [39], i.e., "the internal parameters". $R_{\text {camera }}^{\text {body }}$ is a transformation matrix of the alignment angles between the satellite and the camera, i.e., "the external parameters". $R_{J 2000}^{W G S} 84$ is the transformation matrix from the J2000 coordinate system to the WGS84 coordinate system. $R_{\text {star }}^{J 2000}$ is the attitude transformation matrix from the satellite body coordinate system to the $\mathrm{J} 2000$ coordinate system. $\left[X_{G P S} Y_{G P S} Z_{G P S}\right]_{W G S 84}^{T}$ is the position of the projection center in the WGS84 coordinate system that is obtained by orbit position post-processing technology using the raw GPS data; the post-processing accuracy of ZY-3 is superior to $5 \mathrm{~cm}$. [XYZ $]_{W P S 84}^{T}$ represents the geographic coordinates of the ground object in WGS84.

(2) The step calibration method is adopted for ZY-3's in-flight geometric calibration[40-43].A reference image is simulated with a large-scale DOM/DEM if the satellite attitude and orbit data are given; then, the simulated image is resampled to the same resolution as the ZY-3 image. Phase correlation is employed for the image registered between the ZY-3 image and the simulated reference image to obtain many dense reference points along and across the track of the ZY-3 image [43]. The sequential solution method is used to calculate $\operatorname{tg}\left(\psi_{Y}{ }^{\prime}\right), \operatorname{tg}\left(\psi_{X}{ }^{\prime}\right)$ and $R_{\text {camera }}^{\text {body }}$ with Equation (28). Certainly, if $\left(\operatorname{tg}\left(\psi_{Y}{ }^{\prime}\right), \operatorname{tg}\left(\psi_{X}{ }^{\prime}\right)\right)$ of all CCD are known, then $R_{\text {camera }}^{\text {body }}$ can be directly calibrated by Equation (28) using GCPs.

(3) According to the equivalent relationship between the attitude matrix and the attitude quaternion, along with quaternion theory [44], the difference between the on-board and post-processed attitudes can be calculated by $\Delta q=\bar{q}^{-1} \otimes q$, where $\bar{q}$ denotes the post-processed attitude, and $q$ denotes the on-board attitude. The quaternion deviation $\Delta q$ can be transformed to the Euler angle defined in $\mathrm{J} 2000[45,46] . \delta \lambda \approx 2 q_{1}, \delta \theta \approx 2 q_{2}, \delta \phi \approx 2 q_{3}$.

(4) Collect the ZY-3 stereo images (forward-view, nadir-view and backward-view) that cover the verification site, and obtain the image coordinates of homonymy points in the stereo images by image registration or artificial pricking. Suppose $\left(x_{l_{1}}, y_{l_{1}}\right),\left(x_{m_{1}}, y_{m_{1}}\right)$ and $\left(x_{r_{1}}, y_{r_{1}}\right)$ are the image coordinates of one GCP in the forward-view, nadir-view and backward-view images, respectively. Assume that $\mathrm{x}$ is the flight direction and $\mathrm{y}$ is the CCD direction. For the forward-view image, the attitude and position information of $x_{l_{1}}$ is interpolated with the imaging time, and the CCD detector of $y_{l_{1}}\left[\operatorname{tg}\left(\psi_{Y}{ }^{\prime}\right) \operatorname{tg}\left(\psi_{X}{ }^{\prime}\right)-1\right]_{y}^{T}$ is interpolated by the calibrated internal parameters. Similarly, the attitude and orbit data of this GCP in the other views are also obtained. The object space coordinate $\left[\begin{array}{lll}X^{\prime} & Y^{\prime} & Z^{\prime}\end{array}\right]_{W G S 84}^{T}$ of the GCP can be calculated by Equation (28). The difference between the calculated $\left[\begin{array}{lll}X^{\prime} & Y^{\prime} & Z^{\prime}\end{array}\right]_{W G S 84}^{T}$ and the known $\left[\begin{array}{lll}X & Y & Z\end{array}\right]_{W G S 84}^{T}$ of this GCP is computed, and the RMS of the difference is used to evaluate the accuracy.

\section{Experiment Data and Analysis of the Results}

The internal parameters of the ZY-3 camera had been calibrated before the acquisition of the test data, and the accuracy is as high as 0.2 pixel and varied by 0.03 pixels in the CCD direction for six months according to in-orbit change detection. Therefore, the internal parameters do not need to be calibrated in this study.

Although the external parameters were also calibrated before the test data acquisition, they varied by at least $2 \sim 3$ arc $\mathrm{s}$ for three months, which equates to $5 \sim 8 \mathrm{~m}$ in the position accuracy for the ZY -3 satellite; 
thus, precise external parameter calibration is necessary for obtaining a reasonable attitude precision comparison in this study.

\subsection{Data Preparation}

Three different sites were selected for the experiment. One was used for external parameter calibration, and the other sites were used to validate and compare the on-board and post-processed attitude accuracy. A brief description of the three sites is presented below.

The calibration site used here for ZY-3 is referred to as the Taiyuan Site and is located in the Taiyuan suburb of Shanxi Province. This region covers approximately $500 \mathrm{~km}$ along the track and $50 \mathrm{~km}$ across the track. Ten scenes of image data spanned this region. All of the forward-view, nadir-view, and backward-view image data were used for exterior orientation.

The two validation sites were located in areas with different terrains and were used to analyze and compare the accuracy of the on-board and post-processed attitudes. One test site was located on the Taihang Mountain of Shanxi Province and covered an area of $300 \times 50 \mathrm{~km}$ with an altitude of 150 to $1600 \mathrm{~m}$. The Taihang site can be used to validate the attitude accuracy of the long strip images. The Tianjin site, with an area of approximately $50 \mathrm{~km} \times 50 \mathrm{~km}$, covers several urban areas in Tianjin City. The Tianjin site located in the North China Plain, where the elevation varies within $10 \mathrm{~m}$. This site was used to validate the attitude accuracy of the short strip images. Table 1 contains additional details regarding the test site.

Table 1. Calibration/Validation site and test data.

\begin{tabular}{ccccccc}
\hline Test Site & $\begin{array}{c}\text { Image } \\
\text { Acquisition Date }\end{array}$ & $\begin{array}{c}\text { Track } \\
\text { Number }\end{array}$ & Image Path/Row & $\begin{array}{c}\text { Number of } \\
\text { GCPs }\end{array}$ & Distribution & Terrain \\
\hline $\begin{array}{c}\text { Taiyuan } \\
\text { (calibration) }\end{array}$ & 3 February 2012 & 381 & $\begin{array}{c}\text { Ten scenes: } \\
6 / 120 \sim 6 / 129\end{array}$ & 8 & well-distributed & Mountain \\
\hline Taihang(validation) & 8 February 2012 & 457 & Six scenes: $5 / 120 \sim 5 / 125$ & 36 (checkpoints) & well-distributed & Mountain \\
\hline Tianjin (validation) & 28 February 2012 & 785 & One scene: $896 / 127$ & 22 (checkpoints) & well-distributed & Plain \\
\hline
\end{tabular}

For the suburban area, artificial and natural objects on the ground, such as road intersections, hilltops, and rivers, were selected as GCPs. These objects are clearly shown in the images from any season and are easy to access. For the urban area, artificial objects, such as building corners or marked points on a sports field, were selected as GCPs. The distribution of GCPs is shown in Figure 4. The geographic coordinates of the GCPs were obtained by using a continuously operating reference system (CORS) with a high precision at 3-5 cm, as shown in Figure 5a. The corresponding image coordinates of these GCPs were acquired by artificial pricking, as shown in Figure 5b. These GCPs were used as validated references to evaluate the geometric accuracy of the method that does not use GCPs. 


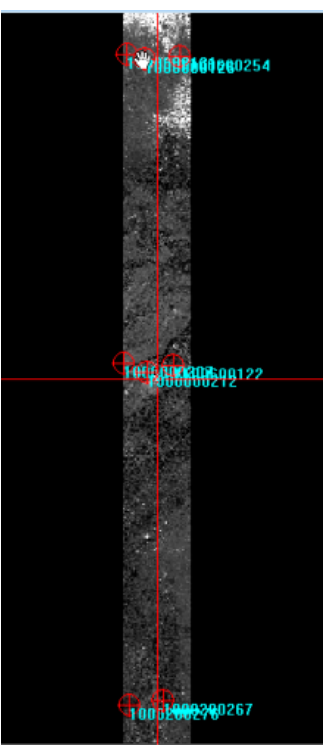

(a)

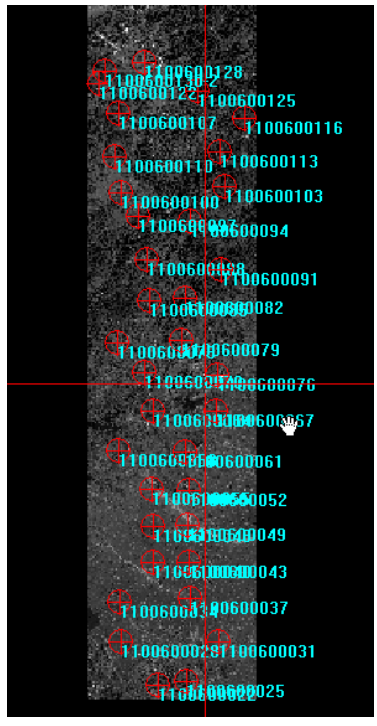

(b)

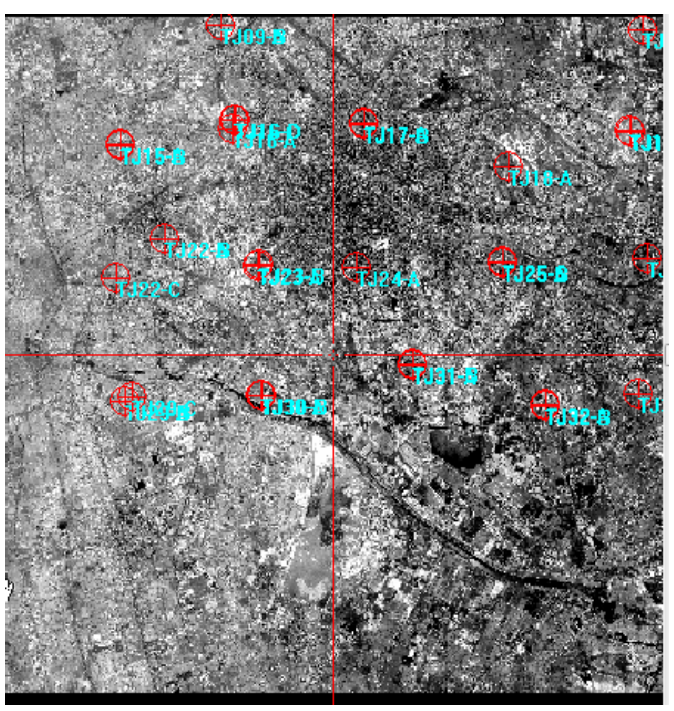

(c)

Figure 4. The distribution of GCPs in Test sites (nadir-view). (a) Taiyuan site (381th track); (b) Taihang site (457th track); (c) Tianjin site (785th track).

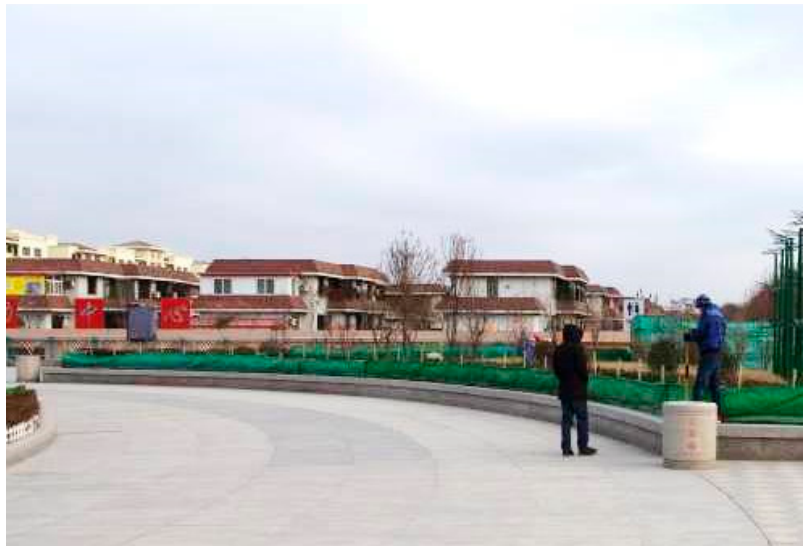

(a)

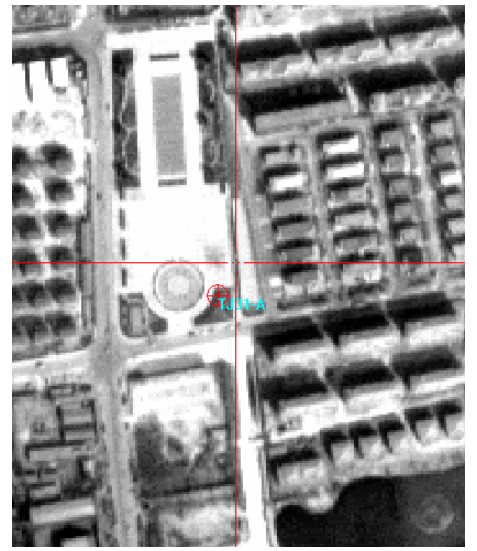

(b)

Figure 5.Ground control point (GCP) acquisition. (a)Field surveying; (b)Artificial pricking (Nadir view).

\subsection{Results and Discussion}

Before attitude accuracy evaluation, the external parameters calibrated by the Taiyuan site were used to correct any systematic geometric errors. Table 2 provides the results of this calibration when using the data from the Taiyuan site, in which BWD, NAD, and FWD are the backward-view, nadir-view, and forward-views of the camera, respectively. $f, w$, and $k$ denote the Euler angles of the three axes using the coordinate system definition in [42]. Table 3 shows the influence of calibration on the positioning accuracy. Note that $X$ and $Y$ are the UTM projection coordinates of latitude and longitude in WGS84, and $Z$ is the ellipsoidal height. The geometric positioning residual results indicate that the interlock angle between the satellite attitude and camera is eliminated, and the random error of the two types of attitude can be evaluated and compared after calibration. 


\subsubsection{The Post-Processed Results and Comparison with the On-Board Attitudes}

The difference between the on-board and post-processed attitudes reflects the accuracy of the deviations. In this study, the raw attitude data corresponding to the image were processed by the system that we developed.

Two sets of raw attitude data from the 457th and 785th tracks were extracted during the same period, and an integrated post attitude determination was implemented. The ground-based post-processed attitude results are shown in Figures 6, where they-axis is the attitude expressed in quaternions, with quaternion vectors of $q_{1}, q_{2}$ and $q_{3}$ in the $\mathrm{J} 2000$ coordinate system. J2000 is the inertial system defined in the celestial sphere [47]. The attitude expressed in quaternion of $y$-axis is dimensionless without any physics meaning. The $x$-axis denotes the accumulated sampling time in seconds since1 January 2009.

Table 2.Exterior orientation result (three panchromatic cameras).

\begin{tabular}{cccc}
\hline \multirow{2}{*}{ Camera } & \multicolumn{3}{c}{ Three Axis Angle (Units: Radian) } \\
\cline { 2 - 4 } & $\boldsymbol{f}$ & $\boldsymbol{w}$ & $\boldsymbol{k}$ \\
\hline BWD & 0.0024873321 & 0.0017746478 & 0.0028587395 \\
NAD & 0.0005171015 & 0.0017320041 & 0.0037815675 \\
FWD & 0.0018654008 & 0.0017649895 & 0.0030016442 \\
\hline
\end{tabular}

Table 3. Position accuracy comparison before and after calibration.

\begin{tabular}{ccccc}
\hline $\begin{array}{c}\text { Geographical } \\
\text { Coordinate }\end{array}$ & ---- & Minimum (m) & Maximum (m) & RMSE (m) \\
\hline \multirow{2}{*}{$X$} & Before calibration & 52.783 & 63.438 & 58.028 \\
& After calibration & 0.227 & 5.714 & 3.355 \\
\hline \multirow{2}{*}{$Y$} & Before calibration & 37.166 & 31.135 & 34.258 \\
& After calibration & 0.071 & 2.818 & 2.031 \\
\hline \multirow{2}{*}{$Z$} & Before calibration & 0.570 & 6.722 & 3.605 \\
& After calibration & -0.142 & -3.446 & 2.165 \\
\hline
\end{tabular}

However, there is a positive correlation between the accuracy of the gyro bias estimations and the accuracy of the attitude filtering results. Figure 7, shows that the gyro bias estimations vary with time in the forward, backward and smoothed filtering processes of 457th track's data, respectively. Figure 8, shows that the gyro bias estimations vary with time in the forward, backward and smoothed filtering processes of 785th track's data, respectively. Obviously, although the estimated gyro bias is divergent at the beginning of forward and backward filtering, it becomes flat and smooth by weighted averaging in the smoothed filtering. It indicates the bias estimation precision was improved after smoothed filtering. The smoothed filtering indirectly improves the attitude accuracy. 


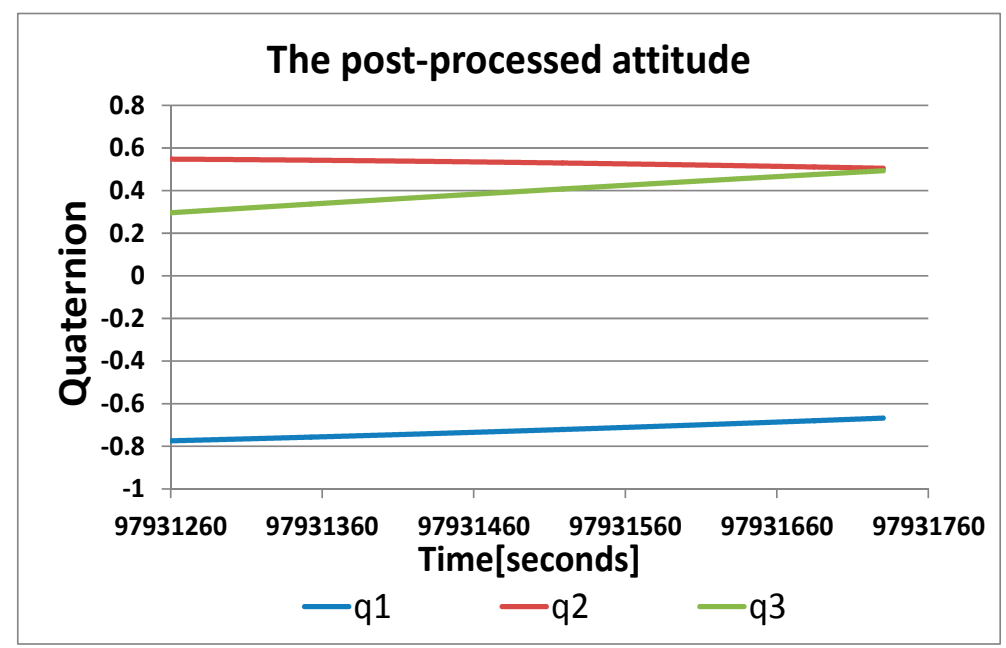

(a)

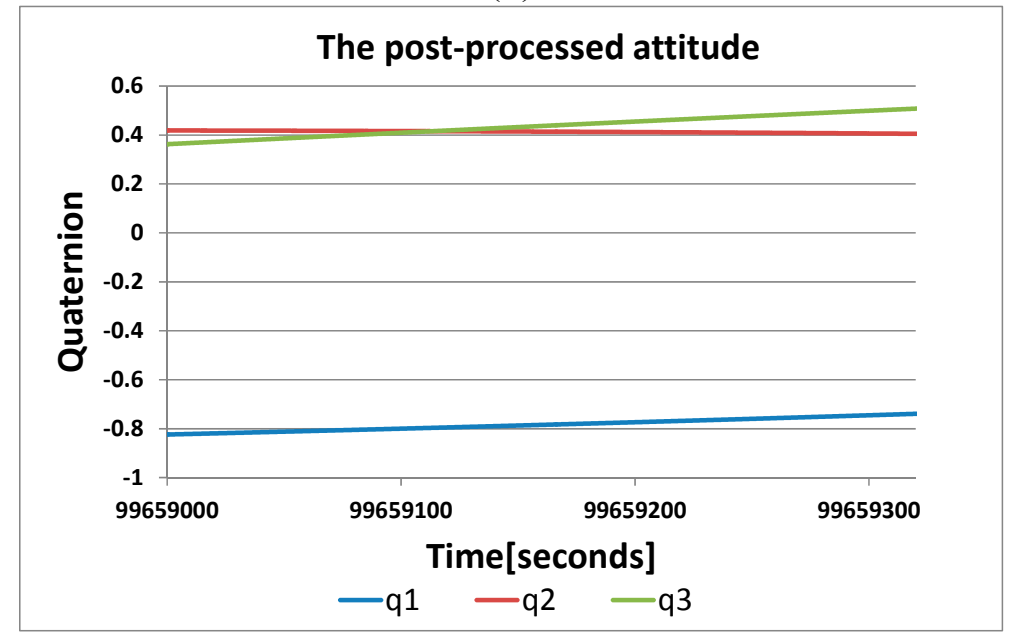

(b)

Figure 6. Post-processed attitude results (quaternion). (a) Post-processed attitude results (the 457th track); (b) post-processed attitude result (the 785th track).

The attitude differences between the on-board and post-processed results are calculated and shown in Figure 9, in which the $x$-axis also denotes the accumulated sample time from 1 January 2009.The $y$-axis denotes the difference between the on-board and post-processed attitude in units of arc seconds.

As seen from Figure 9, the variation of attitude random error with time is very small. The result indicates that this forward-backward filter is very stable and can eliminate the initial vibration in the convergence process, what is impossible with a unidirectional filter. This difference value is less than two arc seconds, which indicates that the difference value between the on-board and post-processed determination is very small, and the post-processed attitude determination approach has proven effective. However, it is difficult to say that which one is better; the accuracy of the on-board and post-processed attitude determinations should be evaluated by geometric positioning method. 


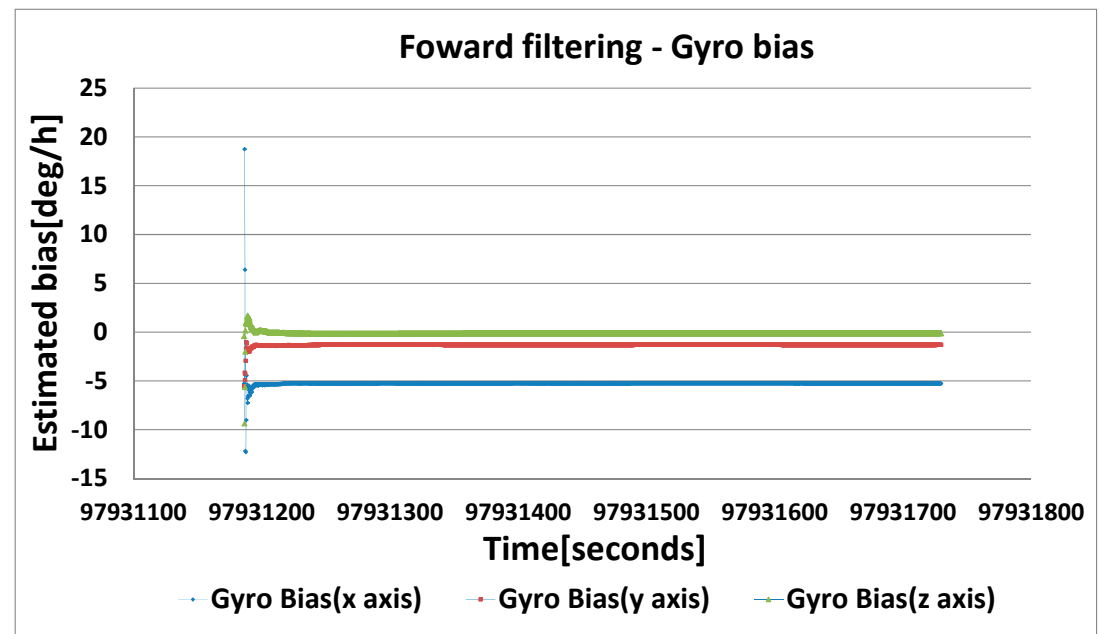

(a)

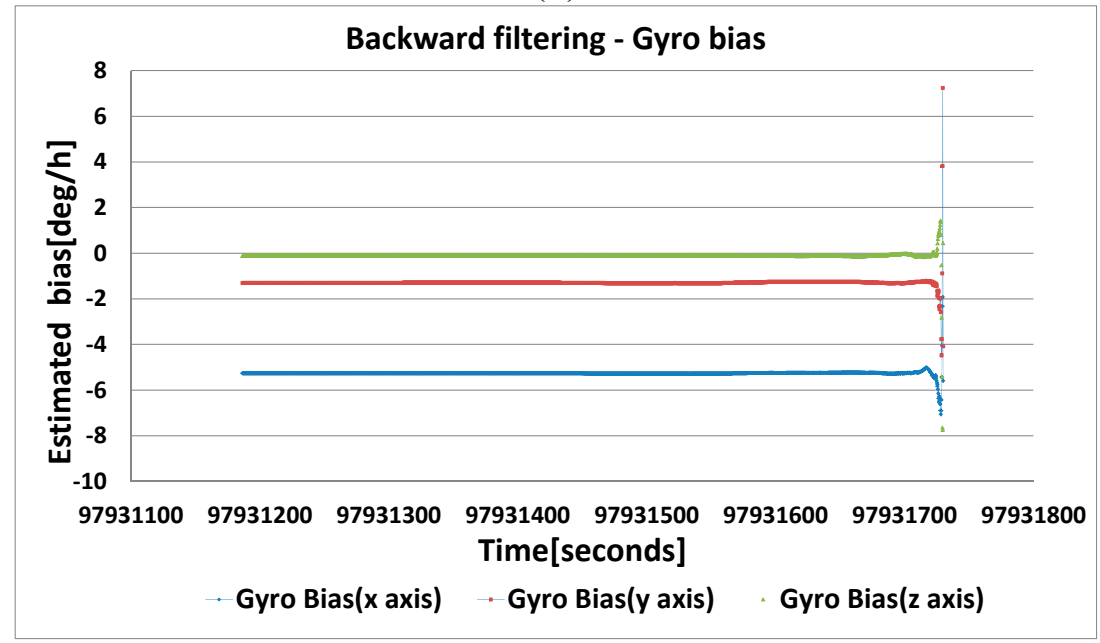

(b)

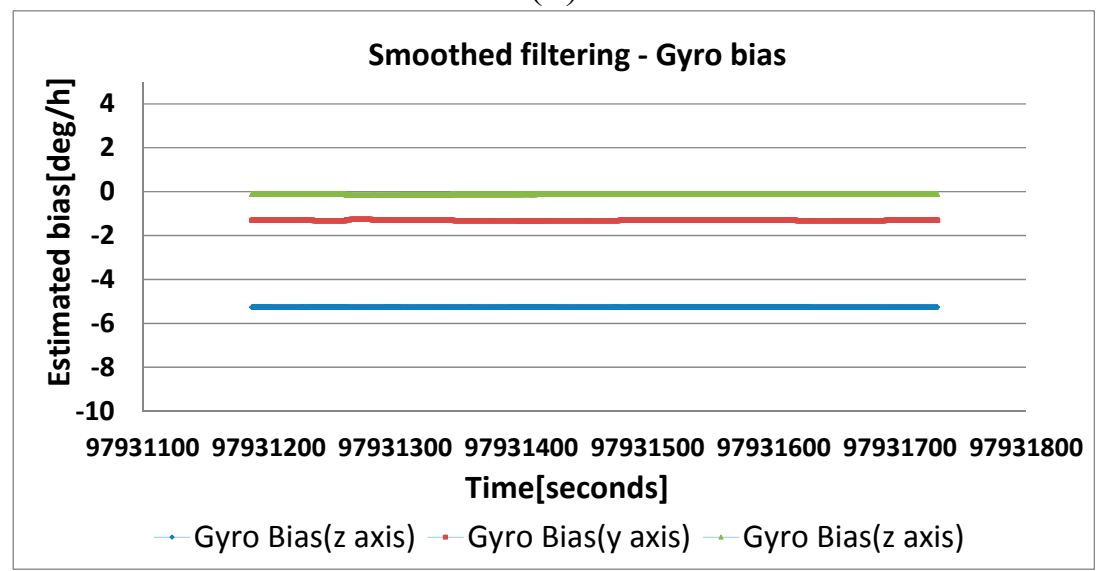

(c)

Figure 7.Post-processed attitude results (quaternion) (the 457th track). (a) The gyro bias of the forward filtering; (b) the gyro bias of backward filtering; (c) The gyro bias of the smoothed filtering. 


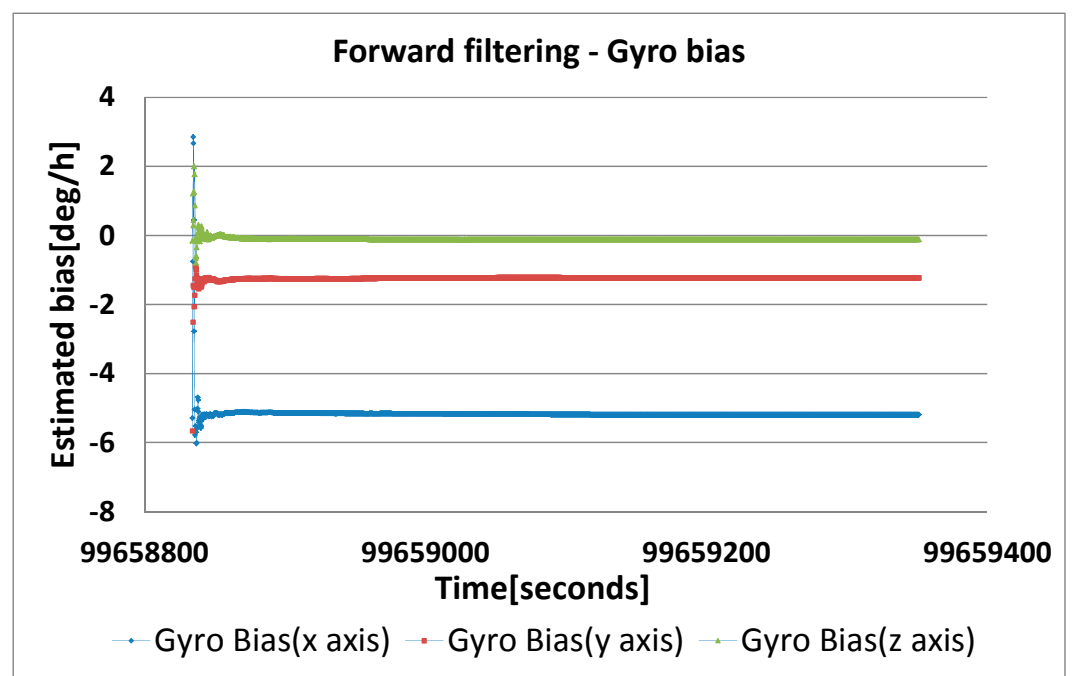

(a)

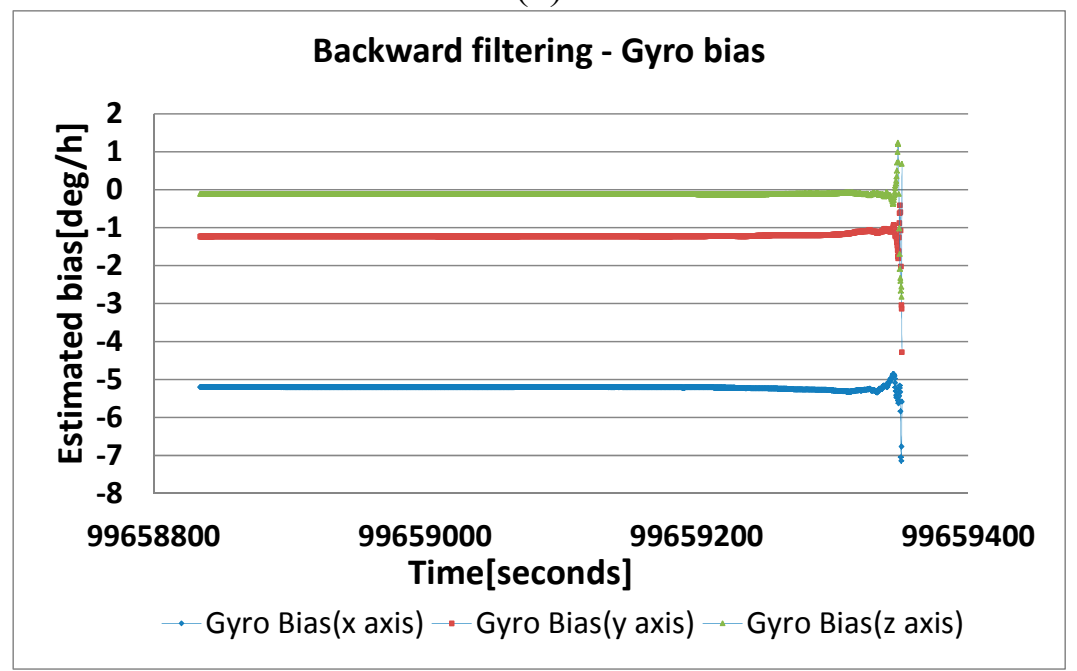

(b)

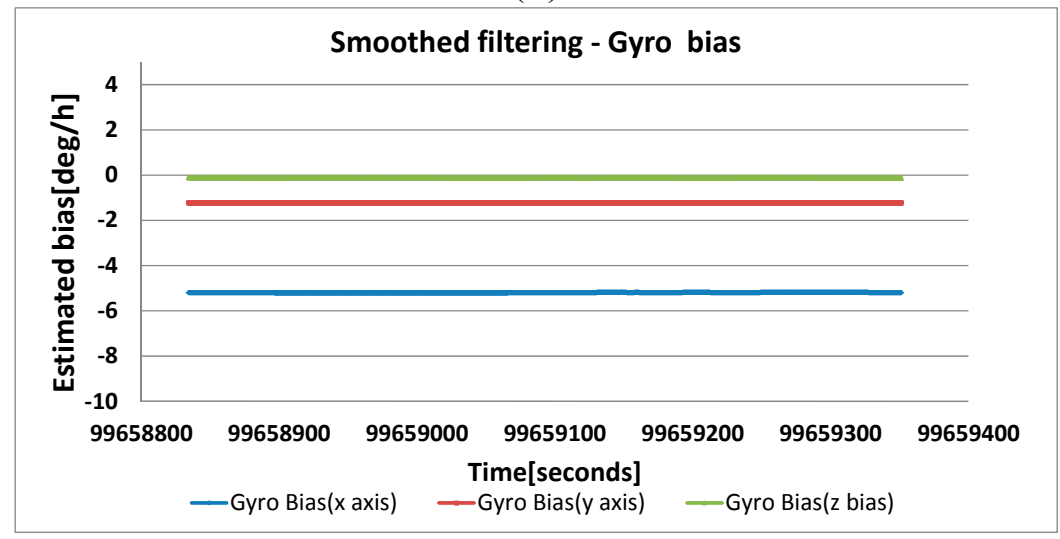

(c)

Figure 8. Post-processed attitude results (quaternion) (the785th track). (a) The gyro bias of forward filtering; (b) the gyro bias of backward filtering; (c) The gyro bias of smoothed filtering. 


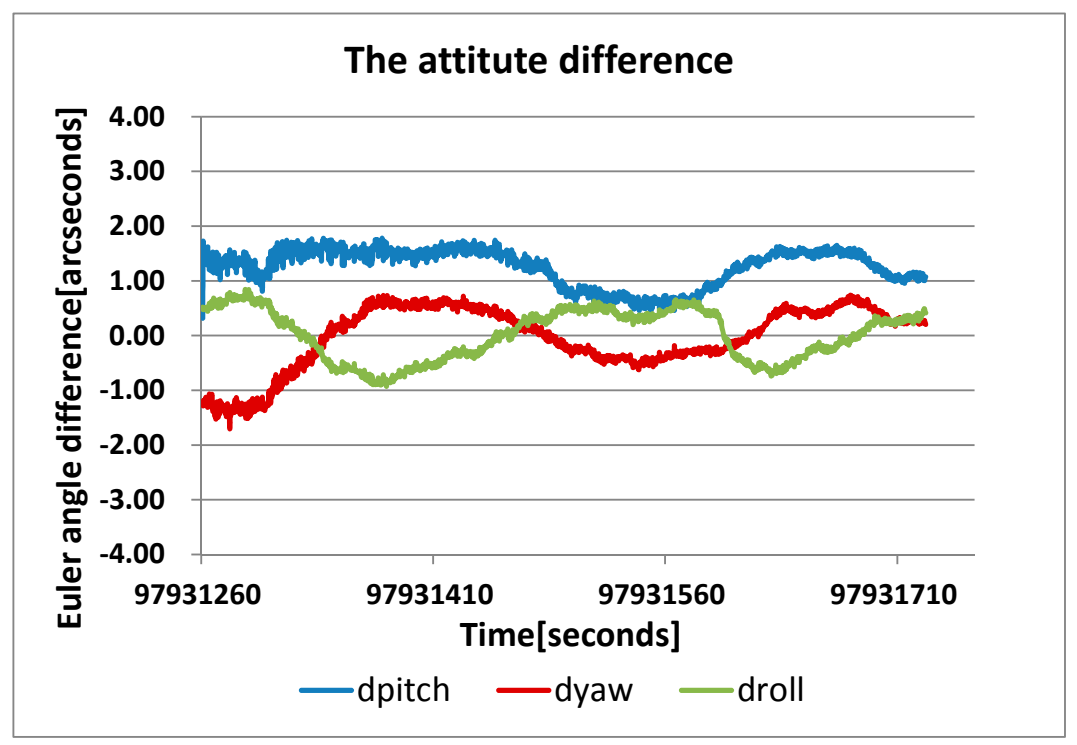

(a)

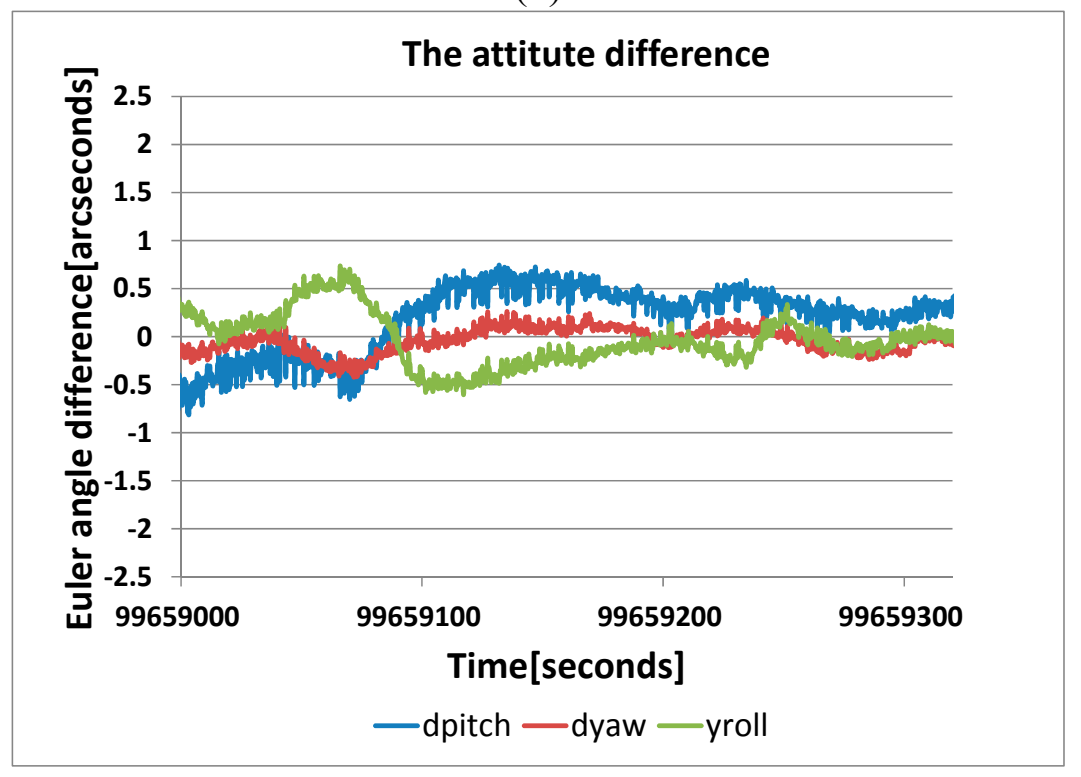

(b)

Figure 9.Attitude difference between the on-board and post-processed results (Euler angle).

(a) Attitude difference (the457th track); (b) attitude difference (the 785th track).

\subsubsection{Evaluation and Comparison of the Attitude Accuracy}

Two methods are employed for evaluating the accuracy of the on-board and post-processed attitude determinations. Both of them can be mutually complementary and verified.

In the first method the GCPs are only taken as reference standards; the geographic coordinates (WGS84) of the reference standards are used for the accuracy evaluation. The reliability of this method is related with the number of the GCPs. However, there is very heavy workload of GCPs' acquisition, so the number of the GCPs is limited. In the second method, the tie points (aka homonymous points) in the overlapping region of the nadir-view, forward-view and backward-view imagery are used for evaluating the attitude accuracy. The free net adjustment takes the least residual error of image points as a constraint condition based on the sensor rigorous imaging model; the attitude accuracy is evaluated by 
the residual error of tie points in the different imagery. The tie points is collected by image matching, more tie points used in this method can reflect the finer distinction of the attitude result.

(1) Comparison of positioning accuracy without using GCPs

The image data that cover the Taihang Mountain Range and Tianjin were used to evaluate the direct geometric positioning accuracy. In the direct positioning process, the GCPs are not used for block adjustment. All GCPs were considered as reference standards for evaluation, and the verification results are shown in Table 4.

Table 4. Position accuracy comparison without using GCPs (in the Earth-centered system). (a) Positioning accuracy comparison without using GCPs in the Taihang Mountain region (the 457th track); (b) Positioning accuracy comparison without using GCPs in the Tianjin area (the 785th track).

\begin{tabular}{ccccc}
\hline $\begin{array}{c}\text { Geographical } \\
\text { Coordinate }\end{array}$ & Attitude & Minimum (m) & Maximum (m) & RMSE (m) \\
\hline \multirow{2}{*}{$X$} & On-board & 4.009 & 12.147 & 8.080 \\
& Post-processed & 2.750 & 11.070 & 7.107 \\
\hline \multirow{2}{*}{$Y$} & On-board & 7.010 & 15.466 & 12.27 \\
& Post-processed & 6.425 & 14.671 & 11.57 \\
\hline \multirow{2}{*}{$Z$} & On-board & 0.729 & 7.315 & 3.633 \\
& Post-processed & 0.138 & 6.290 & 3.631 \\
\hline \multirow{2}{*}{ Geographical } & Attitude & Minimum (m) & Maximum (m) & \multirow{2}{*}{ RMSE (m) } \\
Coordinates & On-board & 9.485 & 13.025 & 11.465 \\
\multirow{2}{*}{$X$} & Post-processed & 8.618 & 11.914 & 10.311 \\
\hline \multirow{2}{*}{$Y$} & On-board & 0.096 & 3.863 & 2.294 \\
& Post-processed & 0.057 & 2.125 & 1.191 \\
\hline \multirow{2}{*}{$Z$} & On-board & 6.903 & 13.117 & 9.796 \\
& Post-processed & 3.517 & 10.728 & 6.796 \\
\hline
\end{tabular}

(b)

As shown in Table 4, the positioning accuracy without using GCPs is very high even at the mountainous Taihang validation site. However, the accuracy of the Tianjin validation site is relatively low in all but they-axis direction because the data acquisition time is closer to the calibration time (only five days apart). Therefore, the variations in the calibration parameters were small.

When other parameters in the rigorous imaging model are constant, such as the position, internal, and external parameters of the camera, the two types of attitude are compared by the positioning accuracy calculated without using GCPs. It is clear that the accuracy of the positioning result that is based on the post-processed attitude is higher. The error of elevation, which is related to the attitude accuracy, can be significantly reduced, and the planimetric accuracy is improved by 1-2 $\mathrm{m}$. In addition, the positioning differences agree with the differences between the on-board and post-processed attitudes, which demonstrates that the post-processed attitude accuracy is better than the on-board accuracy. 
(2) Image point residuals of the tie points

The tie points of the forward-view, backward-view, and nadir-view are acquired by automatic stereo matching. The grid size is set to $9 \times 9$ pixels to speed up the matching process and to distribute the tie points more evenly. Certain large residual points were removed when the auto-matching is completed. Two hundred tie points were generated in the image that covered the Taihang site and 242 tie points were generated in the image that covered the Tianjin site. Free net adjustment was performed with the two sets of attitude data based on the rigorous imaging model. Other parameters in the free net adjustment, such as position and internal and external parameters, remained unchanged.The changes in accuracy were only related to the different sets of attitude data.

The residuals of the tie points from any two of the three views were computed. The statistical results of the residuals for the Taihang and Tianjin images are described in Table 5, respectively. In addition, $X$ and $Y$ are UTM projection coordinates of latitude and longitude in the WGS84, and $X Y$ is the planimetric accuracy (that is, $X Y=\sqrt{X^{2}+Y^{2}}$ ).

Table 5. Image of the point residual statistics of the tie points after free net adjustment. (a) Image point residual statistics of the Taihang image(the 457th track); (b) Image point residual statistics of the Tianjin image(the 785th track).

\begin{tabular}{ccccc}
\hline $\begin{array}{c}\text { Image } \\
\text { Coordinate }\end{array}$ & Attitude & Minimum (Pixel) & Maximum (Pixel) & RMSE (Pixel) \\
\hline \multirow{2}{*}{$X$} & On-board & 0.003 & 2.450 & 0.735 \\
& Post-processed & 0.002 & 2.295 & 0.682 \\
\hline \multirow{2}{*}{$Y$} & On-board & 0.001 & 1.657 & 0.382 \\
& Post-processed & 0.000 & 1.537 & 0.361 \\
\hline \multirow{2}{*}{$X Y$} & On-board & 0.006 & 2.533 & 0.828 \\
& Post-processed & 0.005 & 2.449 & 0.772 \\
\hline
\end{tabular}

(a)

\begin{tabular}{ccccc}
\hline $\begin{array}{c}\text { Image } \\
\text { Coordinate }\end{array}$ & Attitude & Minimum (Pixel) & maximum (Pixel) & RMSE (Pixel) \\
\hline \multirow{2}{*}{$X$} & On-board & 0.019 & 4.097 & 1.706 \\
& Post-processed & 0.004 & 3.501 & 1.113 \\
\hline \multirow{2}{*}{$Y$} & On-board & 0.001 & 3.360 & 0.701 \\
& Post-processed & 0.000 & 3.026 & 0.564 \\
\hline \multirow{2}{*}{$X Y$} & On-board & 0.060 & 4.681 & 1.797 \\
& Post-processed & 0.014 & 4.400 & 1.315 \\
\hline
\end{tabular}

(b)

As shown in Table 5, the range between the minimum and maximum residuals of the tie points using the post-attitude process is less than the on-board range, which indicates that the post-processed attitude stability and accuracy are better than the on-board accuracy, which is also reflected by the Root Mean Square Error (RMSE). 


\section{Conclusions}

Improving the attitude accuracy is one important method for enhancing the level of geometric processing. Based on the raw telemetry star-tracker and gyro data that were accumulated within a given period, we proposed a forward-backward UKF method for post-processing to improve the attitude accuracy. The method adopts the strategy of improving processing accuracy but sacrifices processing time. The proposed method fully uses the characteristics of the abundant computing resources and requires no rapid processing on the ground. In addition, the forward-backward smoother can use attitude observation information for a fixed time interval to determine optimal results by weighted smoothing. Furthermore, this method employs UKF filter algorithms that can perform better for noisesuppression by using an unscented transformation (UT) rather than an EKF in a nonlinear attitude system.

Experiments with the raw attitude data of the ZY-3 satellite were performed to demonstrate the feasibility of the proposed method. The post-processed attitude data were compared with the on-board attitude data using different methods, including attitude difference comparisons, a direct positioning accuracy comparison without using GCPs, and an image point residual comparison of the tie points, which were used to obtain a fair and objective evaluation. The experimental results indicate the following: (1) The filtering accuracy of the proposed algorithm is not affected by the initial value, in contrast to a unidirectional filter;(2) This smoother can eliminate the shock of filtering at the initial phase and avoid attitude results that are unusable in map product production; (3) The proposed algorithm maintains the basic structure of the Unscented Transformation, which can achieve a higher estimation accuracy, as observed from the comparison of the RMS of the tie points' residual and positioning accuracies.

Overall, the proposed method provides a new solution for the post-processing attitude data. The software for the raw star-tracker/gyro data post-processing was developed to provide daily attitude processing services for the ZY-3 satellite after validation. The post-processed attitude is used for different levels of imagery production, which is applied to many industries. In the future, this method should be popularized for other high-resolution remote sensing satellites other than the ZY-3 satellite in China.

\section{Acknowledgments}

The authors thank the editors and the reviewers for their constructive and helpful comments, which led to substantial improvement of this paper. This work is supported by the Natural Science Foundation of China (41301525), the Surveying and Mapping Public Welfare Project of China (No. 201412001), and Project in the National Science \& Technology Pillar Program (2012BAH31B00).

\section{Author Contributions}

All authors have made major and unique contributions. Xinming Tang designed the overall concept of the study; Junfeng Xie implemented the methodology and writed the manuscript; Xiao Wang (also corresponding author) contributed to optimization of the algorithm and helped revise the manuscript; and Wanshou Jiang performed the data validation and comparison analysis in the experiments.

\section{Conflicts of Interest}

The authors declare no conflict of interest. 


\section{References}

1. Dial, G.; Bowen, H.; Gerlach, F.; Grodecki, J.; Oleszczuk, R. IKONOS satellite, imagery, and products. Remote Sens. Environ. 2003, 88, 23-36.

2. Tang, X.; Xie, J.; Zhang, G. Development and status of mapping satellite technology. Spacecr. Recovery Remote Sens. 2012, 33, 17-20.

3. Kalman, R.E. A new approach to linear filtering and prediction problem. J. Basic Eng. 1960, 82, 95-108.

4. Speyer, J. Computation and transmission requirements for a decentralized linear-quadratic-Gaussian control problem. IEEE Trans. Autom. Control. 1979, 24, 266-269.

5. Kerr, T. Decentralized filtering and redundancy management for multi-sensor navigation. IEEE Trans. Aerosp. Electron. Syst. 1987, 23, 83-119.

6. Calson, N.A.; Berarducci, M.P. Federated Kalman filter simulation results. Navigation 1994, 41, 297-321.

7. Anderson, B.D.O.; Moore, J.B. Optimal Filtering; Prentice-Hall: Englewood Cliffs, NJ, USA, 1979.

8. Psiaki, M.L. Attitude determination filtering via extended quaternion estimation. J. Guid. Control Dyn. 2000, 23, 206-214.

9. Rtliter, A.H.J.D.; Damaren, C.J. Extended Kalman Filtering and nonlinear predictive filtering for spacecraft attitude determination. Can. Aeronaut. Space J. 2002, 48, 13-23.

10. Lefebvre, T.; Bruyninckx, H.; Schutter, J.D. Kalman filters for nonlinear systems: A comparison of performance. Int. J. Control. 2004, 77, 639-653.

11. Jerry, M.M. Lessons in Estimation Theory for Signal Processing, Communications and Control; Prentice Hall: Upper Saddle River, NJ, USA, 2005.

12. Kaminski, P.; Bryson, A.; Schmidt, S. Discrete square root filtering: A survey of current techniques. IEEE Trans. Autom. Control. 1971, 16, 727-736.

13. Hammarling, S. A Survey of Numerical Aspects of Plane Rotations. Available online: http://www.maths.manchester.ac.uk/ sven/pubs/mddx.pdf (accessed on 18 November 2014).

14. Wang, H.; Gregory, R. On the reduction of an arbitrary real square matrix to tridiagonal form. Math. Comput. 1964, 87, 501-505.

15. Chandrasekar, J.; Kim, I.S.; Bernstein, D.S.; Ridley, A.J. Cholesky-based reduced-rank Square-Root Kalman filtering. In Proceedings of the 2008 American Control Conference (ACC), Seattle, WA, USA, 11-13 June 2008; pp. 3987-3992.

16. Bierman, G.J. Measurement updating using the U-D factorization. In Proceedings of the 1975 IEEE Conference on Decision and Control, Houston, TX, USA, 10-12 December 1975; pp. 337-346.

17. Julier, S.J.; Uhlmann, J.K. A new extension of the Kalman filter to nonlinear system. In Proceedings of the 11th International Symposium on Aerospacel/Defence Sensing, Simulation and Controls, Orlando, FL, USA, 21 April 1997.

18. Julier, S.J. The scaled unscented transformation. In Proceedings of the 2002 American Control Conference, Anchorage, AK, USA, 8-10 May 2002; pp. 4555-4559.

19. Crassidis, J.L.; Markley, F.L. Unscented filtering for spacecraft attitude estimation. Am. Inst. Aeronaut. Astronaut. 2003, 26, 536-542. 
20. Julier, S.J.; Durrant-whyte, H.F. Process models for the high-speed navigation of road vehicles. In Proceedings of the 1995 International Conference on Robotics and Automation (ICRA), Nagoya, Japan, 21-27 May1995; pp. 101-105.

21. Wan,E.A.; Merve, R. The unscented Kalman filter for nonlinear estimation. In Proceedings of the 2000 IEEE Adaptive Systems for Signal Processing, Communications, and Control Symposium: AS-SPCC, Lake Louise, AB, Canada, 1-4 October 2000; pp. 153-158.

22. Merve, R.;Wan, E.A. The square-root unscented Kalman filter for state and parameter-estimation. In Proceedings of the 2001 International Conference on Acoustics, Speech, and Signal Processing, Salt Lake City, UT, USA, 7-11 May 2001; pp. 3461-3464.

23. Burello, M. SPOT1 In-Orbit Attitude Control System Performances. In Proceedings of the 1987 AAS Guidance and Control Conference, Keystone, CO, USA, 4-8 February 1987.

24. Grodecki, J.; Dial, G. IKONOS geometric accuracy validation. ISPRS Arch. 2002, 34, 50-55.

25. Mulawa, D. On-orbit geometric calibration of the OrbView-3 high resolution imaging satellite. ISPRS Arch. 2004, 35, 1-6.

26. Rauch, H.E.; Tung, F.C.; Striebel, T. Maximum likelihood estimates of linear dynamic system. Am. Inst. Aeronaut. Astronaut. 1965, 80, 1445-1450.

27. Sarkka, S. Unscented rauch-tung-striebel smoother. IEEE Trans. Autom. 2008, 53, 845-849.

28. Takanori, I. Precision attitude and position determination for Advanced Land Observing Satellite (ALOS). Proc. SPIE 2009, 34, 1845-1848.

29. Department of the Interior U.S. Geological Survey. Landsat 7 Image Assessment System (IAS) Geometric Algorithm Theoretical Basis Document. Available online: http://landsat.usgs.gov/ documents/LS-IAS-01_Geometric_ATBD.pdf (accessed on 19 November 2014).

30. Sungkoo, B. GLAS Spacecraft Attitude Determination Using CCD Star Tracker and 3-Axis Gyros. Ph.D. Thesis, the University of Texas at Austin, Austin, TX, USA, 1998.

31. Li, D. China's First Civilian Three-line-array Stereo mapping satellite: ZY-3. Acta. Geod. Cartogr. Sin.2012, 41, 317-322.

32. Tang, X.; Xie, J. Overview of the key technologies for high-resolution satellite mapping. Int. J. Digit. Earth 2012, 5, 227-240.

33. Vallado, D.A. General perturbation techniques. In Fundamentals of Astro dynamics and Applications, 2nd ed.; Kluwer Academic Publishers: Dordrecht, The Netherlands, 2001; pp.707-713.

34. Tapley, B.D.; Schutz, B.E.; Born, G.H. Fundamentals of orbit determination. In Statistical Orbit Determination; Elsevier Academic Press: Waltham, MA, USA, 2004; pp. 159-284.

35. Bar-Itzhack, I.Y.; Harman, R.R. Optimized TRIAD algorithm for attitude determination. Am. Inst. Aeronaut. Astronaut. 1997, 20, 208-210.

36. Spot Image. Available online: http://www-igm.univ-mlv.fr/ riazano/publications/GAEL-P135DOC-001-01-04.pdf (accessed on 19 November 2014).

37. Tang, X.; Zhang, G.; Zhu, X. Triple linear-array image geometry model of ZiYuan-3 surveying satellite and its validation. Acta Geod. Cartogr. Sin. 2012, 4, 33-51.

38. Gao, X.; Tang, X.; Zhang, G.; Zhu, X. The geometric accuracy validation of the ZY-3 mapping satellite. Int. Arch. Photogramm. Remote Sens. Spat. Inf. Sci. 2013, XL-2/W1, 111-115.

39. Torjbörn, W. In-flight calibration of SPOT CCD detector geometry. Photogramm. Eng. Remote Sens. 1992, 58, 1313-1319. 
40. Gachet, R. Spot5 in-flight commissioning: Inner orientation of HRG and HRS Instruments. Int. Arch. Photogramm. Remote Sens. Spat. Inf. Sci.2004, 35, 535-539.

41. Bouillon, A.; Breton, E.; De, L.F.; Gachet, R. SPOT5 HRG and HRS first in-flight geometric quality results. In Proceedings of the 9th International Symposium on Remote Sensing, Aghia Pelagia, Greece, 22-27 September 2002; pp. 212-223.

42. Valorge, C.; Meygret, A.; Lebègue, L.; Henry, P.; Bouillon, A.; Gachet, R.; Breton, E.; Léger, D.; Viallefont, F. 40 years of experience with SPOT in-flight calibration. In Proceedings of the 2003 ISPRS International Workshop on Radiometric and Geometric Calibration, Gulfort, MS, USA, 2-5 December 2003; pp. 119-133.

43. Leprince, S.; Barbot, S.; Ayoub, F.; Avouac, J.P. Automatic and precise orthorectification, coregistration and subpixel correlation of satellite images, application to ground deformation measurements. IEEE Trans. Geosci. Remote Sens.2007, 45, 1529-1558.

44. Markley, F.L.; Cheng, Y.; Crassidis, J.L.; Oshman, Y. Averaging quaternions. Am. Inst. Aeronaut. Astronaut. 2007, 30, 1193-1197.

45. Markley, F.L. Attitude error representations for kalman filtering. Am. Inst. Aeronaut. Astronaut. 2003, 26, 311-317.

46. Karlgaard, C.D.; Schaub, H. Adaptive Huber based filtering using projection statistics: Application to spacecraft attitude estimation. In Proceedings of the 2008 AIAA Guidance, Navigation and Control Conference and Exhibit, Honolulu, HI, USA, 18-21 August 2008.

47. Bona, B.; Canuto, E. Star-Tracker Attitude Measurement Model. Available online: http://www.ladispe.polito.it/corsi/meccatronica/02JHCOR/2011-12/Slides/Star_Tracker.pdf (accessed on 19 November 2014).

(C) 2014 by the authors; licensee MDPI, Basel, Switzerland. This article is an open access article distributed under the terms and conditions of the Creative Commons Attribution license (http://creativecommons.org/licenses/by/4.0/). 